\title{
Thermal deformation of 3U CubeSat in low Earth orbit
}

\author{
Vasily Gorev ${ }^{1, *}$, Anatoly Pelemeshko ${ }^{1}$, Alexander Zadorozhny ${ }^{1}$, and Aleksey Sidorchuk ${ }^{1,2}$ \\ ${ }^{1}$ Novosibirsk State University, 630090, Pirogova str., 2, Novosibirsk, Russia \\ ${ }^{2}$ OKB Fifth Generation Ltd., 630090, Nikolaeva str., 11, Novosibirsk, Russia
}

\begin{abstract}
The impact of uneven heating on a satellite structure in low Earth orbit has been considered using the example of $3 \mathrm{U}$ CubeSat. The calculations showed that the thermal deformation of CubeSat structure in orbit caused a deviation between normals to opposite small satellite sides of about $0.03^{\circ}$. Such a deviation is commensurate with the required satellite pointing accuracy approximately $0.1^{\circ}$ necessary for satellite laser communication. It means that to solve similar problems in the CubeSat designing that require such or better CubeSat pointing accuracy, it is necessary to take into account the expected satellite structure thermal deformation.
\end{abstract}

\section{Introduction}

Novosibirsk State University develops a universal modular platform for nanosatellites compatible with CubeSat. The platform should also support optical (laser) communication. The creation of the positioning system module is one of the most important tasks of the project, since it provides the satellite with pointing accuracy required to operate an optical (laser) communication system. The satellite's pointing accuracy with a rigid laser fixing on its structure should not be worse than $0.1^{\circ}$ to direct the laser beam to the ground receiving station [1].

The deformation of the satellite structure due to temperature variations on its surface during the orbital motion is one of the factors affecting pointing accuracy.

While operating in orbit, CubeSat absorbs radiant energy originating from external sources, and emits thermal radiation from its surface into the surrounding space. Uneven radiation heating leads to uneven satellite heating, which can vary widely depending on its design.

In developing a high-precision pointing system it is necessary to understand the impact of heat fluxes on the satellite's surface while in the Earth's orbit and corresponding deformation on the satellite structure in order to make constructive decisions to minimize or compensate for them. The aim of this article is to study the impact of uneven satellite heating in low Earth orbit (LEO) on structure deformation according to the example set by $3 \mathrm{U}$ CubeSat.

\footnotetext{
"Corresponding author: vasily.gorev@gmail.com
} 


\section{CubeSat pointing and its design}

The positioning system of a modern CubeSat generally contains multiple sensors such as Sun sensors, Earth sensors, horizon sensors and star trackers (Figure 1, a). This article describes the configuration of a satellite directed by the $1 \mathrm{U}$ side to face the Earth's surface. The choice of such a positioning can be convenient for an optical device with a large aperture having an extended dimension in the direction of the observation vector. The laser communication optical receiver may also require the satellite assembly stretched along the Earth's radial direction.

The positioning sensors and laser communication unit (or observation unit) can be fixed to either of opposite small satellite sides.

This means that, due to thermal deformation, there will be an additional angle between the optical axis of the laser communication system (or observation) and the optical axis of the star tracker, as the most accurate of the positioning sensors, which was missed at calibration. Thus, the positioning system will have a systematic error.

For ease of calculation, it is assumed that the orbital motion vector is perpendicular to one of the $3 \mathrm{U}$ sides.
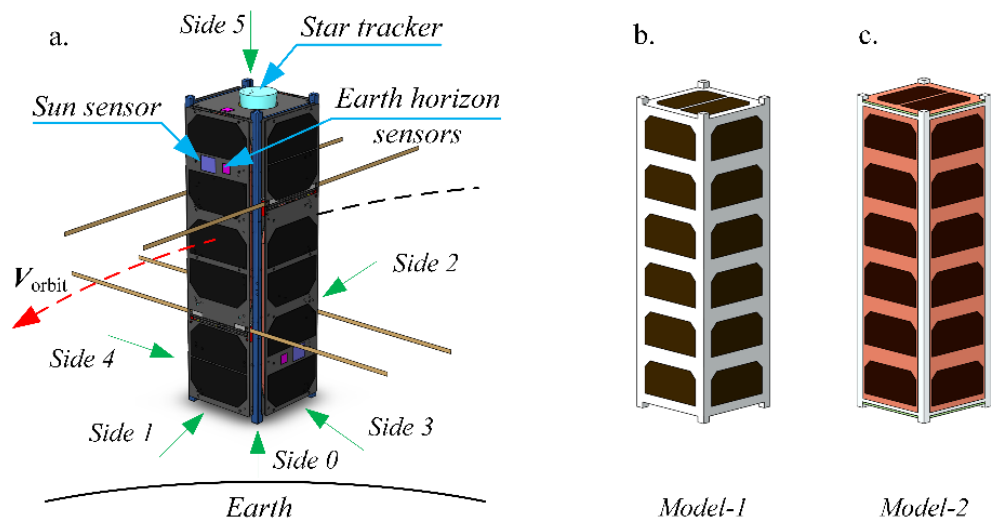

Fig. 1. 3U CubeSat positioning relative to the Earth and the positioning system sensors' configuration on the satellite surface (a). CubeSat structure design (b, c).

This article describes two versions of the $3 \mathrm{U}$ CubeSat structure (Figure 1, b, c). The first simplified model-1 is a solid structure combining the framework and panels made of anodized aluminum alloy 2024. For this alloy, the solar absorption and IR radiation emission coefficients $(\alpha=0.42$ and $\varepsilon=0,15)$ were taken from study [2]. In model-2, the satellite's side panels consist of a $1.6 \mathrm{~mm}$ thick FP-4 glass epoxy, which are coated with a $0.2 \mathrm{~mm}$ copper layer on both sides. In order to compare only the impact of thermal conductivity, the $\alpha$ and $\varepsilon$ coefficients for the model-2 made of FR- 4 are equal to the coefficients of the model-1 made of the alloy 2024.

In each model under study, the photoelectric transducers on the sides of the satellite structure are selected areas on the surface, which are also taken into account in the calculation with their own coefficients $\alpha=0.92$ and $\varepsilon=0.9$. There are no electronic components and printed circuit boards inside the models. 


\section{Thermal regime calculation procedure}

The thermal regime calculation procedure for the satellite is performed using the SolidWorks Simulation CAD tool taking the values of the absorbed radiation power calculated in the MatLab environment as boundary conditions.

Modeling of the CubeSat radiation regime was carried out for circular low Earth orbit. The orbit height $\mathrm{H}$ and the angle between the plane of the satellite's orbit and its direction to the Sun B are the main external parameters of the task [3, 4]. For this calculation, the orbit height $\mathrm{H}$ was assumed to be $450 \mathrm{~km}$, which roughly corresponds to the orbit of the international space station. In order to cover the possible range of angles $\mathrm{B}=0^{\circ}-90^{\circ}$, a family of orbits perpendicular to the ecliptic plane is considered.

The following radiation sources are included in the calculation: solar radiation, Earth albedo and Earth infrared radiation.

Depending on angle B, different thermal regimes of the satellite orbital motion can be observed. Thus, for a satellite with an absolutely black surface, the mean orbital radiation power and mean orbital temperature on its surface are calculated. The satellite is believed to come into thermal equilibrium instantly (Figure 2).

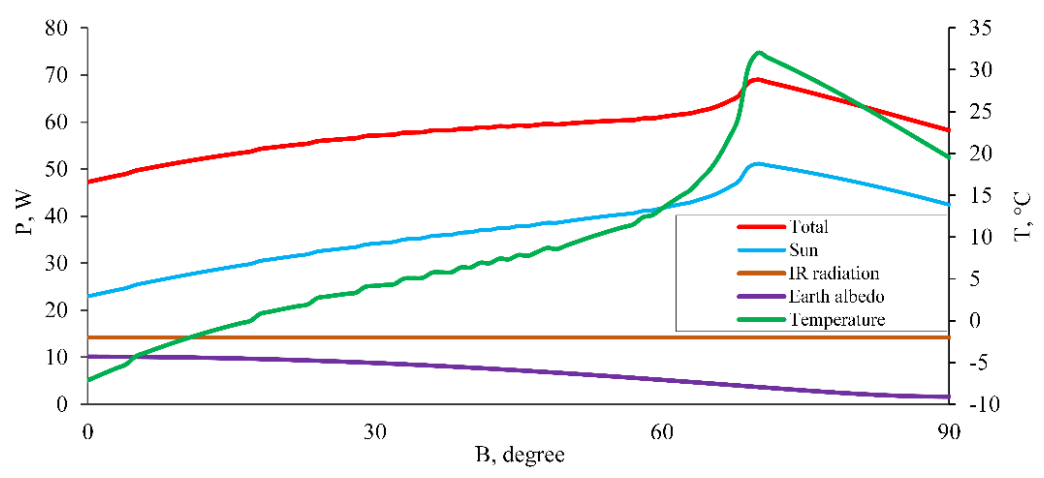

Fig. 2. The equilibrium mean orbital temperature of the satellite with an absolutely black surface (green line) and mean orbital power of the heat flux on different components on the CubeSat surface.

The range of $B$ angles can be divided into two parts. In the subrange $\mathrm{B}=0^{\circ}-70^{\circ}$, the satellite enters the Earth's shadow and, therefore, experiences considerable temperature fluctuations. In the subrange $\mathrm{B}=70^{\circ}-90^{\circ}$, during the entire orbital flight the satellite is illuminated by the Sun, and the mean temperature does not vary widely. The subranges boundary for mean orbital power and temperature is shown on the figure in the form of a tooth at point $\mathrm{B}=70^{\circ}$.

It can be seen that with a given pointing the satellite gets the greatest amount of energy in the orbit corresponding to the angle $\mathrm{B}=70^{\circ}$. When angle $\mathrm{B}$ increases, the amount of energy reaching the satellite surface is reduced. This is due to the satellite pointing and to the change in the Earth's albedo, because if $\mathrm{B}$ is about $90^{\circ}$, the proportion of the Earth albedo in the thermal balance of the satellite is much less than the other components.

Table 1. Distribution of the absorbed power on the $3 \mathrm{U}$ CubeSat sides.

\begin{tabular}{|c|c|c|c|c|c|c|c|}
\hline Side № & $\mathbf{0}$ & 1 & 2 & 3 & 4 & 5 & $\Sigma$ \\
\hline $\boldsymbol{P}_{f v}, \mathrm{~W} / \mathrm{M}^{2}$ & 199.7 & 93 & 93 & 1399.3 & 90.4 & 0 & - \\
\hline$P_{A 2024}, \mathrm{~W} / \mathrm{M}^{2}$ & 34.9 & 16.2 & 16.2 & 612.6 & 15.1 & 0 & - \\
\hline$S_{f v}, \mathrm{M}^{2} * 10^{-3}$ & 5.45 & 16.6 & 16.6 & 16.6 & 16.6 & 5.45 & 77.3 \\
\hline $\boldsymbol{S}_{\boldsymbol{A 2 0 2 4}, \mathrm{M}^{2} * 10^{-3}}$ & 4.55 & 16.37 & 16.37 & 16.37 & 16.37 & 4.55 & 74.6 \\
\hline $\boldsymbol{P}_{\text {total }}, \mathrm{W}$ & 1.25 & 1.81 & 1.81 & 33.26 & 1.75 & $\mathbf{0}$ & 39.9 \\
\hline
\end{tabular}


However, satellite mean orbital temperature in the orbit $\mathrm{B}=70^{\circ}$ and in the orbit $\mathrm{B}=90^{\circ}$ near the terminator does not differ qualitatively and quantitatively. Therefore, to simplify the calculation of thermal deformation, the thermal regime in the orbit at $\mathrm{B}=90^{\circ}$ is considered, which roughly corresponds to the solar-synchronous orbit passing over the terminator line. From the point of view of the satellite thermal regime and, accordingly, for thermal deformation, this formulation of the problem is stationary. In the presented satellite orientation, the main energy flux falls only on one CubeSat side.

The absorbed radiation power surface density for CubeSat surface materials $\left(\boldsymbol{P}_{f v}\right.$ for photoelectric converters, $\boldsymbol{P}_{\boldsymbol{A 2 0 2 4}}$ for anodized aluminum alloy 2024) and the total radiation power absorbed by the satellite sides are presented in Table 1

As was supposed, the absorbed radiation power has a maximum value on the side, which faces the Sun.

\section{Evaluation of satellite structure thermal deformation}

Static analysis of the CubeSat thermal deformation was performed by SolidWorks Simulation software for two models with varying degrees of detail described above.
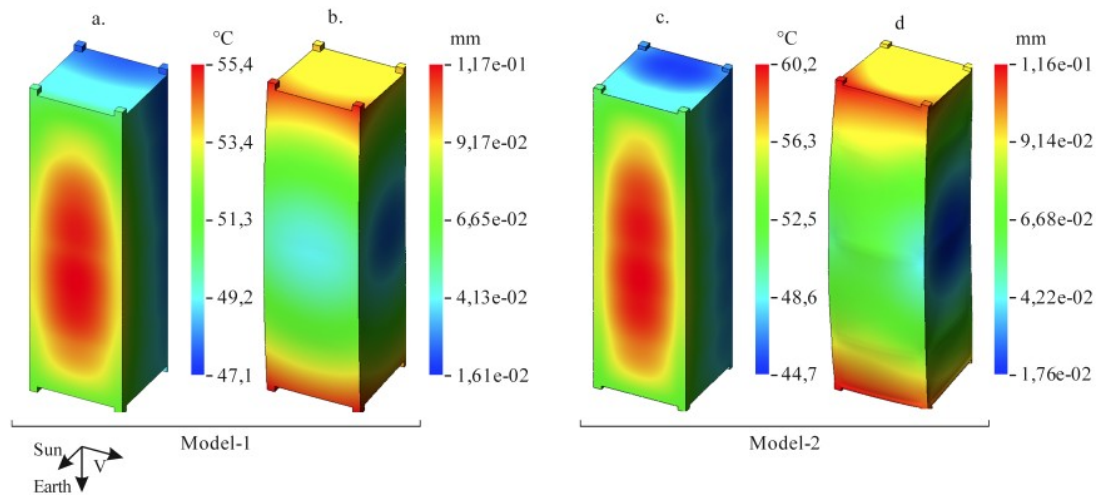

Fig. 3. Basic mode: a, c - surface temperature field, b, d - framework deformation field.

To calculate the displacement field of the structure grid caused by thermal deformation (Figure 3), the results of the thermal calculation for the orbit $\mathrm{B}=90^{\circ}$ were used. The surface temperature difference $\mathrm{dT}=8,3{ }^{\circ} \mathrm{C}$ and $15,5^{\circ} \mathrm{C}$ for the satellite with solid aluminum case and for the satellite made of FR-4, respectively, is explained by the different thermal conductivity of the model-1 and model-2 panels.

It can be seen that specified points on the satellite structure are displaced from their equilibrium positions by more than $0.1 \mathrm{~mm}$. Herewith, the maximum displacements of the model-1 and model-2 points are not qualitatively different, since FR-4 fiberglass composite has a coefficient of thermal deformation comparable with metals.

The satellite construction has a pronounced bend in one direction, because one of its $3 \mathrm{U}$ sides is heated more than others. In addition, the problem has symmetry with respect to the Sun-Earth-satellite plane. In this case it can be asserted that the $1 \mathrm{U}$ sides have much less relative deformation than the heated $3 U$ side, therefore we will consider them approximately flat.

To estimate the angle $\gamma$ between normals to $1 \mathrm{U}$ sides, 4 corner points are selected on the small sides of the layout (Figure 4). For each point, their initial and displaced coordinates in the deformed state were determined. The selected points form four triplets along which it is possible to construct four pairs of vectors directed from each corner of the $1 \mathrm{U}$ side along the $3 \mathrm{U}$ side. For each pair of vectors, a normal was constructed by its cross product. Thus, 
for the opposite small sides, four normal vectors are constructed, and the angles between the corresponding normals of opposite sides are determined.

To solve the pointing problem in which the laser module and the star tracker are fixed on the satellite structure without any compensating technical solutions, the obtained deviation angles will increase the satellite attitude system error. Angular deviation $\left(0.03^{\circ}\right)$ makes a significant part of the required pointing angle accuracy to the receiving station $\left(0.1^{\circ}\right)$

\begin{tabular}{|c|c|c|c|c|c|c|}
\hline \multirow{6}{*}{$\boldsymbol{n}^{\boldsymbol{n}_{b}} \boldsymbol{\Lambda}^{\boldsymbol{n}_{t}}$} & \multicolumn{6}{|c|}{ Model-1 } \\
\hline & Point & 1 & 2 & 3 & 4 & Mean \\
\hline & $\alpha$ & $0,024^{\circ}$ & $0,028^{\circ}$ & $0,028^{\circ}$ & $0,024^{\circ}$ & $0,026^{\circ}$ \\
\hline & \multicolumn{6}{|c|}{ Model-2 } \\
\hline & Point & 1 & 2 & 3 & 4 & Mean \\
\hline & $\alpha$ & $0,030^{\circ}$ & $0,035^{\circ}$ & $0,035^{\circ}$ & $0,030^{\circ}$ & $0,033^{\circ}$ \\
\hline
\end{tabular}

Fig. 4. Scheme for calculating the angle between the normals to the 1U sides. Angular deviation $\gamma$ between the corresponding normal vectors.

Displacement of the specified points due to thermal deformation and the angle between the normals to the $1 \mathrm{U}$ surfaces depends on the difference between the maximum and minimum temperatures over the satellite surface (dT). As the thermal conductivity dT of the structure will decrease, accordingly, the displacement values and the angles between the normals will decrease too.

In designing a nanosatellite, it is necessary to take into account that satellite components thermal deformation can affect the satellite positioning system. A thermal deformation impact on optical system can be reduced by applying a number of technical solutions that make it possible to ensure the constant angles between the optical axes of the optical system units, in particular by the increase in the thermal conductivity of the satellite structure.

\section{Conclusion}

The impact of uneven satellite heating on the satellite structure deformation is analyzed by the $3 \mathrm{U}$ CubeSat in LEO example. It is shown that the thermal deformation of the CubeSat structure, which is in orbit of $450 \mathrm{~km}$ high and angle $\mathrm{B}=90^{\circ}$, causes a deviation of about $0.03^{\circ}$ from the normals to the opposite small sides of the satellite. Such a deviation is commensurate with the required satellite pointing accuracy on the order of $0.1^{\circ}$ necessary for laser communication. Thus, in CubeSat designing to solve problems requiring the stated or better satellite pointing accuracy, it is necessary to take into account the expected satellite structure thermal deformation.

\section{References}

1. S. W. Janson, R. P. Welle, T. S. Rose, D. W. Rowen, D. A. Hinkley, B. S. Hardy, S. D. La Lumondiere, G. A. Maul, N. I. Werner AIAA/USU Conf. SSC15-III-1 (2015)

2. J. L. Golden, NASA Conference Publication 3257, 61 (1992)

3. Space Mission Engineering: The New SMAD Edited by J. R Wertz, D. F. Everett, J. J. Puschell, Microcosm Press, (2011)

4. Spacecraft thermal control handbook. Volume I: Fundamental technologies (Edited by D. G. Gilmore, The Aerospace Press, 2002) 